\title{
ANÁLISIS CLÍNICO: Una remembranza
}

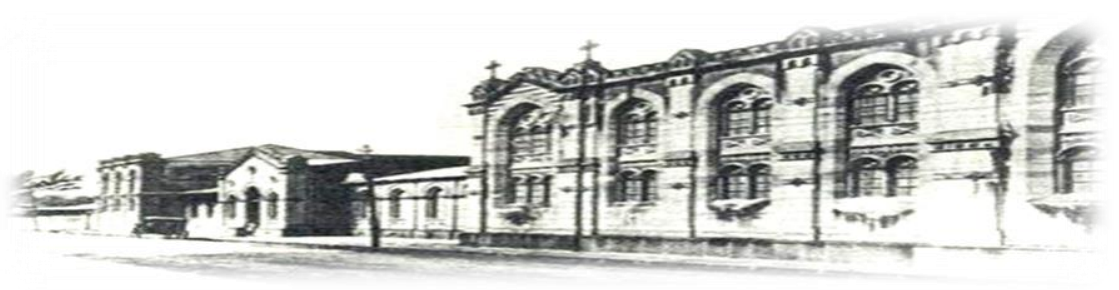

Hospital San Juan de Dios, San José, Costa Rica. Fundado en 1845

$\begin{array}{ll}\text { Recibido: } & 17 / 05 / 2016 \\ \text { Aceptado: } & 15 / 06 / 2016\end{array}$

Luis Fdo. Briceño Rodríguez ${ }^{1}$

${ }^{1}$ Médico Especialista en Medicina Interna. Ex-Miembro Sección de Medicina - Hospital San Juan de Dios. Profesor Asociado de la Universidad de Costa Rica (pensionado). Correo electrónico: $\underline{\text { briceno@racsa.co.cr }}$

\section{RESUMEN}

Recorrido histórico de Análisis Clínico interpretación de la información-, desde sus inicios al presente; disciplina que ha fortalecido la formación universitaria de pregrado, grado $\mathrm{y}$ posgrado médicos.

Se valora la temática de información y formación, y evalúa conceptos actuales sobre la enseñanzaaprendizaje.

Un recuerdo para todos aquellos que hemos forjado nuestra formación académica y/o profesional, completa o en parte en el Hospital San Juan de Dios, entre los años setenta al 2016.

\section{INTRODUCCIÓN}

A principios del segundo lustro de los años setenta del siglo $\mathrm{XX}$, inicié con alegría mi carrera profesional en nuestra Alma Mater, la Universidad de Costa Rica. ¡Qué ilusión embargaba entonces a aquel muchacho que sentía fascinación por todo lo relacionado con la Medicina y por llegar a ser médico!

Poco a poco, y con el suceder de curso tras curso de Ciencias Básicas iba conociendo y entendiendo lo relacionado a la estructura y función de los diferentes tejidos, órganos y sistemas del cuerpo humano, inicialmente en salud y, años después en enfermedad.

Fue en 1977, cuando ingresé por vez primera al sitio que se volvería mi segundo hogar, el "Hospital del Pueblo" -le llamaban-, sí, mi querido Hospital San Juan de Dios (Figura 1).

El "Hospital del Pueblo", aquel que fue baluarte en la atención de quienes padecieron cólera durante la Campaña contra los filibusteros de 1856-1857; aquel que socorrió a personas víctimas del terremoto de Cartago en 1910; aquel que recibió los heridos de la tragedia ferroviaria del Virilla en 1926; aquel hospital que recibía hombres y mujeres sin distingos de clase social. Ese hospital que con 
sus largos corredores, jardines y grandes salones generaba algo especial en quienes llegábamos a él para nuestra formación académica y profesional. El hospital "cuna" de la Medicina y de las ciencias médicas de nuestro país.

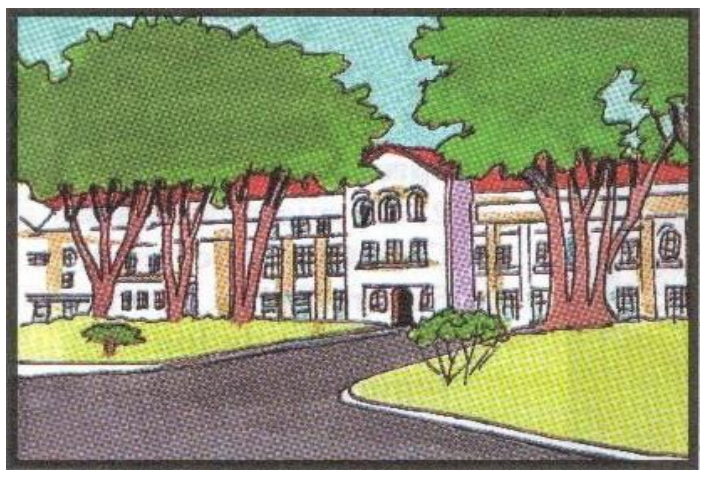

Figura 1: Dibujo del Costado Este del Hospital San Juan de Dios ("Puerta de Médicos").

Dibujo cortesía del Lic. Emilio Dormond Herrera.

Emprendía el camino por el Área Clínica de la Universidad de Costa Rica (UCR) en el Hospital San Juan de Dios (HSJD).

\section{Tercer Año de Carrera}

Durante el primer semestre de 1977 empecé a adquirir las técnicas, destrezas y conocimientos sobre la exploración de los pacientes Semiología/Fisiopatología -, paralelamente al curso de Patología.

Un semestre después, dio inicio lo que con el paso de los años, llegó a convertirse en la especialidad que me apasionaría y daría el saber para encontrar las respuestas a las preguntas que día con día se venían a mi mente. Ese segundo semestre ingresé a mi primer curso de Medicina Interna.

¡Medicina Interna! ¡Qué bello campo en el entendimiento médico! Advertía algo más que un curso universitario.

Preguntas y respuestas, dudas y dudas, más preguntas y más respuestas; y a veces, las respuestas nos llevaban a más dudas y éstas a más preguntas.

Era ir comprendiendo tópico tras tópico el porqué de las enfermedades a que se enfrenta el estudiante y el médico en la atención integral del paciente adulto; pero sobre todo, un curso que iba entusiasmando a aquel muchacho en su vida presente y para su vida futura.

¡Área Clínica!

Ese término iba absorbiendo el interior del aspirante a médico algún día.

\section{Quinto Año de Carrera}

Primer semestre de 1979, ¡qué alegría!; inicié de lleno Medicina Interna II.

Debo indicar que ese año tuve como profesores a dos tutores de rotación clínica quienes practicaban metodologías diferentes de enseñanza. Uno de ellos, nos inculcaba la lectura "del último artículo", y el otro nos enseñaba a "entender el porqué de las cosas".

El primero nos aportaba semanalmente diversos artículos que él había leído, y aún cuando no todo el grupo de rotación comprendiera la totalidad de lo escrito, lo válido era la lectura de ese "último artículo”. ¡Conocíamos medicamentos específicos! ¡Podíamos instruirnos en el tratamiento de enfermedades!

Asimismo, si lo indicado estaba escrito en el Harrison, "estaba escrito en el Harrison" y así debería ser. Además, si el último artículo lo escribía un tal "Dr. Smith, M.D.", en un hospital allende a nuestras fronteras -que nos había dicho el doctor-, así debería ser.

Hoy me pregunto: ¿Era esto enteramente correcto? ¿Cómo llegaríamos a entender en forma plena la enfermedad? ¡Hay tantas enfermedades!

El segundo docente, nos enseñaba a estructurar la información obtenida del paciente órgano por órgano, sistema por sistema.

- ¿Pero el diagnóstico doctor? Preguntaba un estudiante.

- $\quad$ Tenga paciencia, el diagnóstico llegará por su propio peso.

Contestaba el profesor.

Existía diferencia entre un profesor y otro; y en su forma de enseñar. 
Diferentes escuelas.

¡Claro!, comprendí: libertad de Cátedra; piedra angular en una Universidad.

No sé por qué (o tal vez sí) -o fueron cosas del destino -, iba calando más el segundo profesor que el primero; y quizá haya sido que me gustaba más pensar que memorizar. Bueno, qué importancia tiene esa historia de un muchacho que estaba recorriendo su quinto año de carrera de Medicina.

Acá me detendré, y recordaré que ese segundo profesor a quien me refiero, aquel que nos ponía tareas en un cuaderno para ordenar metódicamente los síntomas y signos por órganos y sistemas, y organizar los datos de laboratorio y gabinete por órganos y sistemas, fue el doctor Eduardo Castro Murillo (1928-2010).

El doctor Castro nos hablaba acerca de la "Estructuración de la Información".

- Dr. Castro. Decía otro estudiante.

- $\quad$ Si joven. Respondía EL MAESTRO.

- Una vez estructurada la información por órganos y sistemas: ¿qué?

La visión y opinión del doctor Eduardo Castro Murillo sobre la docencia y la enseñanza de Medicina siempre fueron críticas, respetuosas y acertadas.

Para él (me platicó un día) ${ }^{3,9}$ :

"La docencia no consiste únicamente en la transmisión del conocimiento. La adquisición del conocimiento se puede lograr a través de los libros, de las revistas médicas, de Internet, etc. La docencia es lograr la motivación para descubrir la belleza que hay en la ciencia, en el conocimiento de la Medicina; porque realmente ese conocimiento encierra belleza" 7.

¡Motivación!, excelente concepto. El doctor era un verdadero motivador para todos los que tuvimos la dicha de ser sus alumnos.

\section{Internado Universitario}

Año 1980, ¡qué maravilla! Ya éramos Internos Universitarios, vestíamos gabacha blanca y algunos lucían los estetoscopios alrededor del cuello. Nos llamaban "doctores" (aunque no lo fuéramos aún, pero ya nos llamaban así).

Para entonces, sabíamos que la estructuración de la información por órganos y sistemas que se nos había inculcado nos llevaría a tener una visión global del paciente y nos adiestraba en el dominio de la información. Era la Fase Organizativa de Análisis Clínico, que nos facilitaría establecer el compromiso estructural y funcional, reconocer la presencia de síndromes, determinar la naturaleza del proceso anátomo-patológico y elaborar un resumen sistematizado (un retrato detallado y preciso del paciente): la caracterización del caso, conclusión firme de la Fase Interpretativa.

La siguiente, la Fase Analítica (análisis propiamente dicho), mediante la cual establecemos una estrategia diagnóstica, sea sindrómica 6, 7 , etiopatogénica ${ }^{5,7}$ o anátomo-patológica ${ }^{4,7}$, todas ellas con la elaboración racionalizada de hipótesis.

\section{Residencia Médica}

Las enseñanzas del doctor Castro me estimularon a realizar mi Residencia en Medicina Interna, la cual llevé a cabo en el Hospital San Juan de Dios entre 1982 y 1986.

El doctor Castro Murillo siempre inició los resúmenes de las Sesiones Anátomo-Clínicas de la Sección de Medicina con una caracterización del caso que se iba a analizar, y en muchas oportunidades asignaba la presentación de las sesiones a los Residentes mayores, como impulso en su formación.

\section{Médico Asistente}

A su lado, y dedicándome desde un principio a la docencia en la Sección (por deferencia del doctor Castro), la vida me permitió forjar más y más -ya como Asistente Especialista- este enfoque didáctico en la práctica y enseñanza de la Medicina (Figura 2).

Ambos, durante muchos años y en forma periódica, visitábamos, en los Servicios de la Sección, a Médicos Residentes e Internos Universitarios con 
el propósito de discutir y analizar diferentes casos ingresados, como práctica académica.

Análisis Clínico es entender al paciente, es seguir el método científico en la labor del médico y destreza del estudiante.

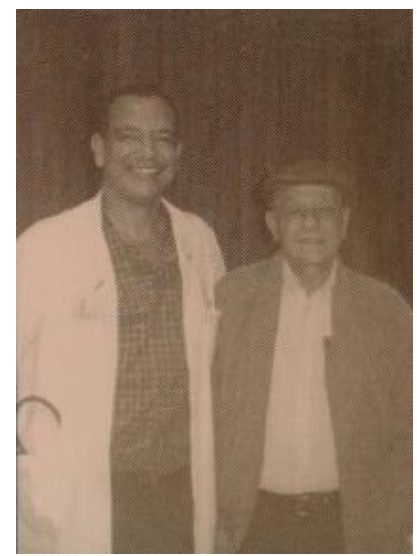

Figura 2. Dr. LF. Briceño y Dr. E. Castro M.

"El aprendizaje de la Medicina es un proceso continuo que se inicia desde nuestra época de estudiantes, a nivel universitario, y que finaliza con el ocaso de nuestras vidas. Nunca dejamos de aprender y comprender algo nuevo" 1 .

En este aprendizaje, información y formación son esenciales. Debe existir un balance entre ambas. $\mathrm{La}$ primera -la información-, importante en la adquisición adecuada, progresiva y actualizada del conocimiento médico, en la incorporación de nuevos conceptos a nuestro saber; y acá, "el último artículo" demanda comprensión e interpretación correctas de esa información. No es sólo información por información.

La segunda -la formación-, requiere el conocimiento detallado de estructura y función, disciplina para entender qué le ocurre al paciente (fisiopatológicamente hablando), comprender cada dato que de él obtenemos, y que pronto nos llevará con mayor certeza al diagnóstico de su enfermedad, y así, nos facilitará un manejo clínico y tratamiento acertados.

Análisis Clínico nos ayudaba en el día a día de nuestro quehacer.

Con los años -y el consejo de los maestrosentendía más, que no hay que ser un enciclopedista. La Medicina no puede ser enciclopedista. La información es tan extensa que hay que conocer cómo se obtuvo y, más importante aún, cómo se interpretó.

La formación efectiva en Medicina Interna es un cambio de actitud hacia la interpretación de la información y la comprensión pormenorizada de esta.

Algunos consideran que se aprende tan sólo con su asistencia a conferencias magistrales en seminarios y congresos, pero olvidan lo indispensable del autodidactismo ${ }^{10}$. Ese aprendizaje con el deseo de explorar por sí mismos el porqué de las cosas.

Todo es parte de una verdadera motivación profesional.

Nuestra formación está al pie de la cama, al lado de nuestros enfermos.

No basta información y conocimiento de las múltiples enfermedades, de extensas citas bibliográficas literales. Debemos comprender integralmente al enfermo y la enfermedad.

Muchos buscan al paciente en el libro, pero no es correcto; se debe buscar el libro en el paciente.

Debemos aprender a investigar, a perder el temor a no encontrar el enfermo en los textos; a perder el temor a decir no sé, ya que muchas patologías las vamos conociendo con el tiempo.

\section{En el curso universitario}

Comprendía que así como el doctor Castro compartió conmigo Análisis Clínico, también debía yo hacerlo con mis alumnos, y permitirme conocerles en su manera de pensar.

Habiéndoseme nombrado Coordinador de la Cátedra de Medicina de la Universidad de Costa Rica en el Hospital San Juan de Dios, establecí en 1996 Análisis Clínico como parte de los cursos semestrales de Medicina Interna I (ME-3005) y Medicina Interna II (ME-5000). Llevaba a cabo con regocijo cada una de las sesiones con los estudiantes. Ya con anterioridad lo estaba realizando en sesiones de análisis con los Internos Universitarios (ME-6004) y con los Médicos Residentes (Posgrado) en la Sección. 
Cada sesión era una aventura mental, en la cual no había preguntas tontas, todas las preguntas eran válidas en el conocer "qué pasa con el paciente".

¡Qué estimulante era ver disfrutar a los muchachos! Era un verdadero juego de aprendizaje. Sí, ¡había motivación!

Todos los alumnos participaban, no sólo en la presentación de los casos clínicos, sino durante la discusión y análisis. Cada quien externaba sus apreciaciones -cualesquiera que fueran-.

\section{Publicación del libro}

Estando el doctor Castro retirado de sus labores, y encontrándose hospitalizado por un quebranto en su salud, me le acerqué y le dije: "doctor, le propongo un proyecto, la publicación de un libro sobre Análisis Clínico" (ya que de él lo había aprendido). Sus ojos seniles se tornaron con un brillo diferente y en su cara visualicé alegría por lo que le proponía.

En 2007, publicamos -a partir de mis varios escritos y de sus acertadas recomendacionesMETODOLOGÍA DE ANÁLISIS CLÍNICO-Un enfoque didáctico en la enseñanza y práctica de la medicina ${ }^{7}$, libro que surca hoy en día las aulas, pasillos y salones de nuestro querido Hospital San Juan de Dios y de otros hospitales del país, a nivel de estudios de pregrado, grado y posgrado universitarios (Figura 3).

«El Análisis Clínico es el análisis integral y cuidadoso de toda la información que se obtiene del paciente. Practicar constantemente el análisis de la información nos lleva a adquirir una destreza intelectual de gran valor y nos hace mejores médicos, porque con frecuencia nos señala que debemos adquirir más conocimientos; o bien, modificarlos y enriquecerlos. Sir William Osler lo dejó dicho hace mucho tiempo: "El mejor aprendizaje lo ofrece el paciente mismo". El Análisis Clínico propone un método sencillo, que indica los pasos a seguir, ordenadamente, para llegar a una interpretación correcta de la información, la cual tiene que ser forzosamente anátomo-fisiopatológica, ya que cada enfermedad tiene su propia expresión estructural y funcional.» 7.

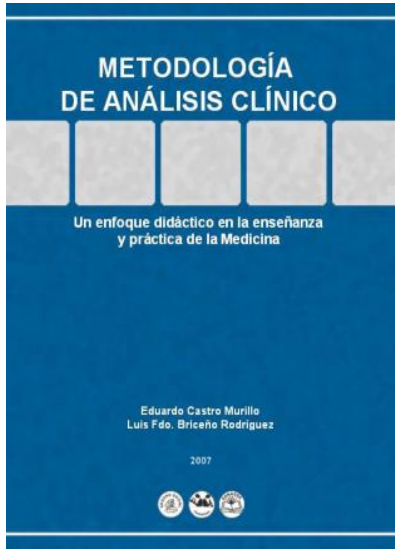

Figura 3. Portada del libro.

\section{Un equipo académico}

Con una mentalidad de compartir el conocimiento y trabajo, decidí a partir de 2009, incorporar como compañeros de curso a dos de aquellos muchachos que en sus años iniciales de carrera habían sobresalido, no sólo por su forma de pensar y capacidad de analizar los casos, sino por el entusiasmo sincero que mostraban durante las diferentes sesiones.

Fue así, como llamé a mis ex-alumnos, en ese entonces médicos Asistentes Especialistas de la Sección de Medicina, doctores Gustavo Vindas Angulo y Freddy Henríquez Varela para que me acompañaran en el curso universitario.

Con alegría accedieron a ello.

La tranquilidad de uno, junto a la hiperquinesia del otro, y el entusiasmo intelectual y docente de ambos, ejercían un balance interesante con su profesor.

Ninguno me defraudó.

Conformamos un equipo de estudio y trabajo docente que hacía nuestras sesiones inolvidables para todos los que en el aula nos encontrábamos (Figura 4). 


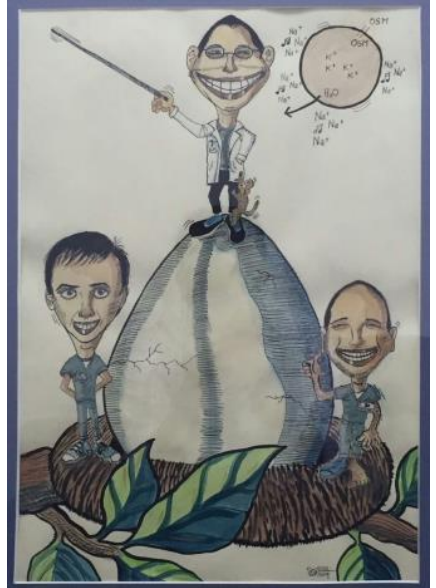

Figura 4. Caricatura realizada por los estudiantes de V Año de Medicina de la UCR / 2011. Título: "Pongan el huevo" (expresión utilizada en el curso para indicar que se debe concretizar una idea.). En su orden -de izquierda a derecha- los doctores F. Henríquez, LF. Briceño y G. Vindas.

Fotografía tomada al original por el Dr. Paolo Duarte S.

¿Qué agradable era ver a los estudiantes aprender la disciplina que el doctor Castro un día nos enseñó!

Ahora nos tocaba a nosotros hacerlo.

Años después comuniqué a Gustavo y a Freddy: "Análisis Clínico es algo de nuestro Hospital y deseo que cuando yo no esté, ustedes dos sigan con el entusiasmo que tienen. Testaré el libro ante el editorial para que ambos continúen" (así lo hice legalmente).

No pensaba aún en los azares del destino.

\section{Una partida no prevista}

A inicios de 2013, circunstancias propias de la vida, me llevaron a jubilarme.

La actividad académico-docente fue, es y será siempre una actividad gratificante en nuestro ser y deber. Aprender a aprender, aprender a enseñar, aprender a partir.

Me alegra haber vivido Análisis Clínico en el aula universitaria y en " $m i$ " Servicio de Medicina Interna que tanto quise y tanto quiero; así como en el Posgrado de Medicina Interna (SEP/CENDEISSS) al que tanto trabajo y esmero dediqué por muchos, muchos años; y me satisface sobremanera haber dejado en manos de quienes dejé el curso que establecí en la Cátedra de la UCR (HSJD).

Así también, ha sido un halago, conocer que las Sesiones de los Residentes e Internos en la Sección continúan como parte de su actividad académica, guiados a partir de 2015 por el doctor Daniel Murillo Castro.

Gustavo Vindas y Freddy Henríquez, así como muchos de los Asistentes jóvenes que me han sucedido, y los Médicos Residentes de la Sección de Medicina, lo han logrado y lo seguirán logrando con excelencia y pasión (estoy seguro de ello).

\section{En la Escuela de Medicina-UCR}

La vida me ha puesto vicisitudes en el camino, pero también me ha regalado satisfacciones. De toda circunstancia se aprende y se continúa adelante.

Las satisfacciones son bellas emociones que nos invitan a vivir; aún cuando, el partir no es triste si algo se ha legado.

Cuando un profesional -en el campo que sea- se dedica a la docencia, no hay nada más grato que conocer que "el alumno supera al maestro". Es un sentimiento del deber cumplido y que la misión que se ha ejercido, ha sido en verdad recompensada.

Los colegas Freddy Henríquez Varela, Carlos Quesada Aguilar y Daniel Murillo Castro, han llevado Análisis Clínico más allá. Los discípulos y compañeros en nuestra época hospitalaria (y amigos en el presente), han establecido el curso "Análisis Clínico Integral" (ME-0323) en las instalaciones del propio campus universitario, a partir de este 2016, como curso optativo de la Escuela de Medicina, para estudiantes de todos los hospitales.

¿Qué complacencia! El curso que inició en nuestro Benemérito "Hospital del Pueblo" ha salido de sus paredes.

La calidad profesional de todos ellos (incluyendo a "Tavo" en el HSJD), son una garantía de que los futuros médicos aprovecharán sus enseñanzas.

La estima, afecto y respeto que siempre me brindaron, es por mí correspondida.

Han emprendido "el viaje fantástico" (LFBR) ${ }^{7}$. 
Me he sentido verdaderamente honrado por su labor e iniciativa. También así se habría sentido el doctor Castro.

\section{Una ciencia - una disciplina}

Ciencia es definida como el conjunto de conocimientos obtenidos mediante la observación y mediante el razonamiento sistemáticamente estructurado, de los que se deducen principios y leyes generales.

La Medicina es ciencia, y como tal es una disciplina exquisita para practicar el método científico.

Ser observadores, realizarse preguntas y buscar las respuestas a las mismas, es una tarea de todo médico y un deber el compartirlo con los alumnos.

La sabiduría radica en comprender. Mantener la mente abierta es una virtud. No todo lo que está escrito es cierto y no todo lo que es cierto está aún- escrito.

Medicina Interna es más que un cuerpo de conocimientos, es una manera de pensar, es una actitud profesional, es alcanzar el dominio de la información y dirigirnos hacia un rumbo seguro.

\section{Enseñanza-aprendizaje}

Los profesores no sólo deben conocer y entender la Medicina, sino que deben ser capaces de explicarla. Compartir lo que nuestra profesión nos ha entregado es un estímulo.

Interpretando lo indicado en el capítulo «El aprendizaje», del libro "EL PRECIO DE LA INTELIGENCIA - La evolución de la mente y sus consecuencias" ${ }^{8}$, sumado a lo que manifesté en el artículo "El aprendizaje de la Medicina" (Revista Práctica Clínica UCR - 1996) ${ }^{1}$, y de lo que considero en la presente publicación, realizo un análisis sobre: transmisión de información y formación propiamente.

El aprendizaje implica dos participantes: un emisor -profesor- y un receptor-alumno-, junto a lo que se transmite: conocimiento, actitud, manera de ver las cosas y forma de pensar (entre otras).

La transmisión puede realizarse por exposición, estimulación, instrucción y colaboración.
La exposición no involucra más que el emisor muestre la información en un "formato cerrado" 8 (p. ej. un texto de un libro, una colección de artículos de otros, ideas y opiniones de otros). El expositor es simplemente un comunicador y el estudiante es un individuo pasivo. La transmisión de la información es meramente unidireccional: docente al alumno.

En la estimulación, el profesor favorece la motivación del estudiante.

En la instrucción y la estimulación, el docente está obligado a interpretar, abstraer y priorizar la información; qué se transmite y cómo se transmite esa información al alumno.

Durante la estimulación, la instrucción y la colaboración, profesor y estudiante son sujetos activos, donde la trasferencia de conocimientos es bidireccional: del docente al alumno y del alumno al docente. Ambos -en principio- disfrutan la actividad y aprenden en la actividad.

En la enseñanza-aprendizaje "la mayor parte de las tareas que se realizan no se aprenden ni se enseñan hablando, sino haciéndolas" 8.

Debemos comprender, razonar, vivir lo bello de la incertidumbre, lo bello de la duda; aprender a vivir la Medicina ${ }^{1}$.

\section{CONCLUSIONES}

Termino con el siguiente párrafo escrito por Ann Druyan en el Capítulo «La casa en llamas» del libro "EL MUNDO Y SUS DEMONIOS - La ciencia como una luz en la oscuridad" (de C. Sagan) ${ }^{2}$ :

"Dorothy Rich, una innovadora profesora de Yonkers, Nueva York, opina que, más importante que los temas académicos específicos, es la formación de capacidades clave, que según ella se incluyen en la siguiente lista: «confianza, perseverancia, atención, trabajo en equipo, sentido común y resolución de problemas». A lo que Druyan- añadiría pensamiento escéptico y capacidad de asombro."

Carl Sagan, en ese mismo libro indica: 
“...toda pregunta es un clamor por entender el mundo." 2

Para aquel muchacho de entonces han pasado los años. Esta remembranza ha sido para mí enriquecedora

“La vida me enseñó a darle respuesta a mis pensamientos y pensamiento a mis respuestas" (LFBR).

17 de mayo de 2016.

BIBLIOGRAFÍA (por año de publicación)

1. Briceño R., LF. El aprendizaje de la Medicina. Práctica Clínica. 1996, 1: 2. (Revista Oficial Sociedad Científica de Estudiantes de Medicina de la Universidad de Costa Rica 1996-).

2. Sagan, C. El mundo y sus demonios - La ciencia como una luz en la oscuridad. Editorial Planeta, 2000. Barcelona, España.

3. Briceño R., LF. Al maestro Eduardo Castro Murillo. Gaceta Médica de Costa Rica. 2004; 6 (1): 13-15.

4. Briceño R., LF. Análisis Clínico en Medicina Interna. Un enfoque didáctico. Gaceta Médica de Costa Rica. 2005; 7 (2): 60-64.

5. Briceño R., LF; Quesada A, CI. Análisis Clínico en Medicina Interna. Razonamiento sobre una base etiopatogénica. Gaceta Médica de Costa Rica. 2006; 8 (1): 22-28.

6. Briceño R., LF; Henríquez V., F. Análisis Clínico en Medicina Interna. Orientación sindrómica. Gaceta Médica de Costa Rica. 2006; 8 (2): 57-60.

7. Castro M., E.; Briceño R., LF. Metodología de Análisis Clínico - Un enfoque didáctico en la enseñanza y práctica de la Medicina. Editorial EDNASSS, 2007. San José, Costa Rica.

8. Agustí, J.; Bufill, E., Mosquera, M. El precio de la inteligencia - La evolución de la mente y sus consecuencias. Editorial Crítica, 2012. Barcelona, España.

9. Briceño R., LF. Reseña biográfica del Dr. Eduardo Castro Murillo. Revista Clínica de la Escuela de Medicina UCR - HSJD. 2013; 3 (7): 30-32.

10. Briceño R., LF. Aprendamos a aprender Medicina. Revista Clínica de la Escuela de Medicina UCR - HSJD. 2014; 4 (3): 44-45.

\section{CONFLICTO DE INTERÉS Y/O AGRADECIMIENTOS}

El autor declara que no existió ningún conflicto de interés en el presente reporte. 